\section{Idiopathic post-operative biliary duct system dilatation; potential etiology and management}

\author{
Mohamed M Nasr ${ }^{1 *}$ and Maged M Nasr ${ }^{2}$ \\ ${ }^{1}$ Consultant, Endoscopic \& General Surgeon, King Fahd Hospital, Saudi Arabia \\ ${ }^{2}$ Consultant, Endoscopic \& Gynecology Surgeon, King Fahd Hospital, Saudi Arabia
}

\section{Summary}

What is called "idiopathic biliary duct system dilatation" or better to name it "un-explained biliary dilatation" is mostly following surgical procedures related to upper gastrointestinal and hepato-bilio-pancreatic systems. Having such situation, adaptive physiology of the biliary duct system has to be considered and rational has to be explained. The vast multimodal progress in techniques of investigations that has been applied on studying the hepato-bilio-pancreatic system has been utilized to clear the clinical ambiguity of biliary duct system dilatation for no logic reason but missing the correlation between both fields; the technical and the clinical ones. This clinical review is trying to fill this gap and introduce a comprehensive discussion of the subject. Mechanical, biochemical and immune causes constitute a wide diversity of etiology related to biliary system dilatation that in some situations is really difficult to verify clinically. On the least, even we could not verify the etiology we need to identify that reaching a closed road is different than postulating suspicions that never exist. This review is a trial collecting all subject-related data that might be related to etiology mechanisms and utilize to find a correlation rationale. At some point verification of such correlation is really a far target that might be even impossible clinically with availing technical tools and hope in the future could be achieved.

\section{More Information}

*Address for Correspondence: Mohamed M Nasr, Consultant Endoscopic \& General Surgeon, Ministry of Health, King Fahd Hospital, Saudi Arabia, Tel: +966 543606 109; Email: drmdnasr@gmail.com

\author{
Submitted: 12 July 2019 \\ Approved: 19 July 2019 \\ Published: 22 July 2019
}

How to cite this article: Nasr MM, Nasr MM. Idiopathic post-operative biliary duct system dilatation; potential etiology and management. Arch Surg Clin Res. 2019; 3: 050-052.

DOI: dx.doi.org/10.29328/journal.ascr.1001032

Copyright: (c) 2019 Nasr MM, et al. This is an open access article distributed under the Creative Commons Attribution License, which permits unrestricted use, distribution, and reproduction in any medium, provided the original work is properly cited

Check for updates

\section{Introduction}

It is a well-known fact that post cholecystectomy biliary changes include biliary duct system dilatation (BD). With the increasing diversity of upper GIT surgeries especially with bariatric ones, the same observation is noticed with no definite causing etiology. In this clinical review, we are going to dig in to the related anatomy, physiology, pathology and clinical aspects.

\section{Definitions}

1. Anatomical definition of common bile duct dilatation (CBDD): There is some debate on the mean diameter of a normal common bile duct (CBD), and studies suggest that the normal range is 4 to $6 \mathrm{~mm}$. In the studies reviewed, a CBD diameter $>7 \mathrm{~mm}$ was considered abnormal [1].

2. Idiopathic biliary duct system dilatation: That is dilated system that could not be related to direct etiology.

Causes of Biliary Dilatation

1. Congenital BD that might be discovered in later life and has specific guidelines for diagnosis [2].
2. Surgical consequence of the following procedures:

a) Post-cholecystectomy: there is increased flow of diluted bile volume that might reach $500 \mathrm{ml}$ instead of $50 \mathrm{ml}$ (increment by ten folds). This change is well known to increase the intra-ductal pressure of the CBD causing dilatation and in addition reversed bile flow. BD may occur at any time but typically occur at a median of 2 years after cholecystectomy. In their series, Park et al. have documented $24.4 \%$ and $29 \%$ of post-cholecystectomy cases showed CBDD more than $7 \mathrm{~mm}$ at 6 and 12 months respectively and all were asymptomatic [3]. Radiological presentations are similar to those with primary or secondary choledocho-lithiasis [4].

b) Post-upper gastrointestinal tract and bariatric surgeries: These all result in CBDD dilation, with changes more pronounced after Roeux-en-Y Gastric Bypass (RYGB). Biliary dilation occurs irrespective of cholecystectomy status. Such procedures are definitely interfering with the secretory mechanism of bile causing derangement and congestion of bile in the sinusoidal and ductal system [5].

1. Drug induced BD $[6,7]$. 


\section{Opiates:}

a) Long usage (initiate sphincter spasm or ambullary achalasia).

b) Cholestatic drugs:

$\neg$ Antibiotics are known incriminated (Cloxacillin, Nafcillin, Fluxacillin, Erythromycin, Travofloxacin, Levofloxacin, Ampicillin) all are cholestatic and last three are hepato-toxic in addition.

$\neg$ Benzo-furans derivatives (Anti-arrhythmic, e.g. Amiodarone).

$\neg$ Benzene-acetic derivatives (anti-inflammatory, e.g. Diclofenac).

2. Independent systemic inflammatory response, sepsis, immune response induction [6].

\section{Bile acids' stagnation trigger two pathways:}

a) Hepatocellular cytokine system activation that causes neutrophil chemotaxis elevating serum liver enzymes as marker.

b) Strike inflammatory response and innate immunity.

3. Idiopathic or un-explained BD: This category is better to be named "BD of unrevealed etiology" as the more we dig investigating the more knowledge we discover. So, etiology revealence of such category is hopefully imminent.

So, we may conclude the following mechanisms that may explain non- obstructive biliary system dilatation:

1. Mechanical etiology.

a. Increment of bile volume and flow rate.

b. Reversed bile flow dynamics that again may cause stagnation and irritation to hepatocytes up to the cyto-toxic effect.

c. Sphincter of Oddi hypertrophy, hypertonia, spasm, achalasia.

2. Hepatocellular, biliary cyto-toxic reaction or combined etiology.

Assessment and diagnosis of the biliary system pathology techniques:

A: Anatomical assessment using the following techniques:

1. Endo-Ultra-Sound (EUS). EUS is a low invasive technique, with high accuracy, that could play a double cost-effective role: identifying pathologic conditions with dismal prognosis, in asymptomatic patients with negative prior imaging tests, and excluding pathologic conditions and further follow-up in healthy subjects [8].

2. Compressed Sensing (CS) Three Dimensional Mag- netic Resonance Cholangio-Pacereaticogram (3-D MRCP). This has showed superior quality information regarding BD in $42.5 \%$ less time than conventional 3D MRCP $[9,10]$.

3. Three Dimensional Balanced Turbo-Field-Echo (BTFE): While MR Cholangio-Pancreatography (MRCP) can only visualize the bile ducts, 3D balanced turbo-field-echo (BTFE) sequence clearly depicts the bile ducts and portal veins as well as drip infusion CT cholangiography (DICCT), without contrast media. Similar to DIC-CT, the BTFE MR sequence had high diagnostic accuracy regarding the branching pattern of the intrahepatic bile duct, especially for the supra- / infra-portal type [11].

4. Dynamic biliary flow (DBF): non-pharmacologic cine-dynamic MR Cholangio-Pancreatography with a Spatially Selective Inversion Recovery Pulse (SSIRP) allows direct and non-invasive visualization of bile flow in the extrahepatic bile duct, demonstrating that reversed bile flow is a physiologic phenomenon [12]. As this assessment technology is relatively recent, and the interpretation with its' application is going on progress, there is still lack of related data and data processing leaving the big question that is "limits of reversed bile flow to consider a pathologic phenomenon rather than a physiologic one".

\section{B: Biochemical Assessment:}

This assessment is crucial in defining the criteria for BD to categorize under idiopathic through exclusion of other pathology induced BD.

\section{Discussion and Conclusion}

Biliary system investigation utilizing the available advanced technologies and the continuously erupting ones have all revealed hidden facts about physiology and dynamics of such system. Biliary criteria as; constitution, volume, pressure, flow dynamics and its secondary effects on the hepato-cellular and pancreatic systems used to be not clearly understood. Current research investigating the hepato-biliopancreatic system as a unit and the highly complicated physical and chemical aspects of the biliary sinusoidal and ductal system, all are gradually clearing up, diverting what used to be named "idiopathic or un-explained biliary dilatation" to a condition under evaluation till reaching a fully understood module.

The so called "idiopathic biliary duct system dilatation" or better to name it "un-explained biliary dilatation" is mostly following surgical procedures related to upper gastrointestinal and hepato-bilio-pancreatic systems. Having such situation, adaptive physiology of the biliary duct system has to be considered and rational has to be explained. Utilizing sophisticated investigation tools available including; Endo-Ultra-Sound, Compressed Sensing Three Dimensional Magnetic Resonance Cholangio-Pacereaticogram (3-D MRCP), Three Dimentional Balanced Turbo-Field-Echo and Non-pharma- 
cologic Cine-Dynamic Magnetic Resonance Cholangio-Pancreato-graphy with a Spatially Selective Inversion Recovery Pulse, all have enlightened us about much of the hidden criteria inside the biliary duct system and revealed details that seem to be provide persuading answers to the unresolved dilemma of such biliary dilatation category for decades. This clinical review, is a trial to resolve most of the ambiguity related to the subject of un-explained biliary duct system dilatation. Upon doing so, we have collected stratified data, validating and selecting the relevant ones and correlating them to the unrevealed questions trying to get logic answers for.

Regarding cholecystectomy surgery, post-operatively the increased flow of diluted bile is well known to increase the intra-ductal pressure of the common bile duct causing dilatation and reversed flow [3]. Similarly, upper gastrointestinal surgeries are interfering with the secretory mechanism of bile causing derangement and congestion of bile in the sinusoidal and ductal system. As a result of this, the Sphincter of Oddi has to counter the increased intraductal biliary pressure and adapt by muscular hypertrophy and hypertonia possibly up to pathological achalasia of the sphincter that adds on the biliary duct dilatation problem progressively with time [5]. In addition to this mechanical etiology, surgical procedures on the gastro-duodenal part, including bariatric surgeries, eventually derange the hormonal control on biliary secretion, interfering with the normal physiology and might play, again, a crucial role in biliary duct system dilatation. Moreover, as a secondary effect to this BD and reversed biliary flow and biliary stagnation exposing hepatic cells to bile acids initiating cyto-toxic effect and triggering elevation of serum hepatic enzymes is another module. Such module of pathology, might resemble other biliary dilatation categories and create a clinical diagnostic difficulty. Verifying such explanations and frame them as a definitive answering module for the condition of biliary duct system dilatation is a research dilemma that needs proofs and might not be resolved for long. Considering such correlation between the clinical diagnosis of post-operative $\mathrm{BD}$ and related physiological adaptation changes may at least release the need to dig for a hidden pathology and prolonged investigations that definitely trouble the patient who is mostly not a patient and exhaust the health service for no rational behind.

Upon postulating these answers, another question is raising; do we have to achieve a routine prophylactic Oddi sphincterotomy to avoid the imminent problem of postoperative biliary duct system dilatation? Is it acceptable to carry on the potential operative and post-operative complications for such sphincterotomy procedure? This would be another clinical dilemma that could hardly reach a commonly agreed answer.

Finally, we emphasize that the aim for this clinical review is to explore and suggest answers that are not easy to verify, for the wondering problem of un-explained post-operative biliary duct system dilatation. By doing so we might release the clinical tension of searching hidden serious problems that mostly are not due.

\section{References}

1. Smith I, Monkemuller K, Wilcox CM. Incidentally Identified Common Bile Duct Dilatation A Systematic Review of Evaluation, Causes, and Outcome. J Clin Gastroenterol. 2015; 49: 810-815.

PubMed: https://www.ncbi.nlm.nih.gov/pubmed/26302495

2. Ishibashi $H$, Shimada $M$, Kamisawa $T$, Fujii $H$, Hamada $Y$, et al. Japanese clinical practice guidelines for congenital biliary dilatation. J Hepatobiliary Pancreat Sci. 2017; 24: 1-16.

PubMed: https://www.ncbi.nlm.nih.gov/pubmed/28111910

3. Park SM, Kim WS, Bae IH, Kim JH, Ryu DH, et al. Common bile duct dilatation after cholecystectomy: a one-year prospective study. J Korean Surg Soc. 2012; 83: 97-101.

PubMed: https://www.ncbi.nlm.nih.gov/pubmed/22880184

4. Coss A, Enns R. The investigation of unexplained biliary dilatation. Curr Gastroenterol Rep. 2009; 11: 155-159.

PubMed: https://www.ncbi.nlm.nih.gov/pubmed/19281704

5. Mehta N, Strong AT, Stevens T, El-HayekK, Nelson A, et al. Common bile duct dilation after bariatric surgery. Surg Endosc. 2019;33: 2531-2538. PubMed: https://www.ncbi.nlm.nih.gov/pubmed/30353239

6. Sharanek A, Burban A, Ciriaci N, Guillouzo A. Pro-inflammatory cytokines enhance dilatation of bile canaliculi caused by cholestatic antibiotics. Toxicol In Vitro. 2019; 58: 51-59.

PubMed: https://www.ncbi.nlm.nih.gov/pubmed/30876886

7. Burban A, Sharanek A, Hüe R, Gay M, Routier S, et al. Penicillinaseresistant antibiotics induce non-immune-mediated cholestasis through HSP27 activation associated with PKC/P38 and PI3K/AKT signalng pathways. Sci Rep. 2017; 7: 1815.

PubMed: https://www.ncbi.nlm.nih.gov/pubmed/28500348

8. De Angelis C, Marietti M, Bruno M, Pellicano R, Rizzetto M. Endoscopic ultrasound in common bile duct dilatation with normal liver enzymes. World J Gastrointest Endosc. 2015; 7: 799-805.

PubMed: https://www.ncbi.nlm.nih.gov/pmc/articles/PMC4501970/

9. Kwon H, Reid S, Kim D, Lee S, Cho J, et al. Diagnosing common bile duct obstruction: comparison of image quality and diagnostic performance of three-dimensional magnetic resonance cholangiopancreatography with and without compressed sensing. Abdom Radiol. 2018; 43: 2255-2261.

PubMed: https://www.ncbi.nlm.nih.gov/pubmed/29302736

10. Hollingsworth KG. Reducing acquisition time in clinical MRI by data under sampling and compressed sensing reconstruction. Phys Med Biol. 2015; 60: 297-322.

PubMed: https://www.ncbi.nlm.nih.gov/pubmed/26448064

11. Ogawa M, Ozawa $Y$, Ohta $K$, Sekiguchi T, Omata S, et al. Usefulness of 3D balanced turbo-field-echo MR sequence evaluating the branching pattern of the intrahepatic bile ducts: comparison with drip infusion CT cholangiography. Abdom Radiol. 2017; 42: 1888-1895. PubMed: https://www.ncbi.nlm.nih.gov/pubmed/28260170

12. Ito K, Kanki A, Yamamoto A, Tamada T, Yasokawa K, et al. Assessment of physiologic bile flow in the extrahepatic bile duct with Cine-Dynamic MR Cholangiopancreatography and a Spatially Selective InversionRecovery Pulse. Radiology. 2014; 270: 777-783.

PubMed: https://www.ncbi.nlm.nih.gov/pubmed/24475829 